\section{A Fast-Marching Time-Domain Layered Finite-Element Reduction-Recovery Method for High-Frequency VLSI Design}

\author{
Houle Gan and Dan Jiao
}

step permitted by the TD-LAFE-RR method is small. To overcome this problem, we propose a fast-marching TD-LAFE-RR method in this work. This method increases the time step of the TD-LAFE-RR method by three orders of magnitude. Meanwhile, it maintains the same computational efficiency of the TD-LAFE-RR scheme. In what follows, we first analyze the time step allowed by the LAFE-RR method in solving general multilayered structures; then delineate the proposed fast-matching method.

\section{InVESTIGATION OF THE TIME STEP ALlOWED BY THE TIME-DOMAIN LAFE-RR METHOD}

The electric field $\mathbf{E}$ inside a 3D integrated circuit satisfies the secondorder vector wave equation

$$
\begin{aligned}
\nabla \times\left[\mu_{r}^{-1} \nabla \times \mathbf{E}(\mathbf{r}, t)\right] & +\mu_{0} \varepsilon \partial_{t}^{2} \mathbf{E}(\mathbf{r}, t) \\
& +\mu_{0} \sigma \partial_{t} \mathbf{E}(\mathbf{r}, t)=-\mu_{0} \partial_{t} \mathbf{J}(\mathbf{r}, t) \text { in } V
\end{aligned}
$$

subject to certain boundary conditions. In (1), $\mu_{r}, \mu_{0}, \varepsilon, \sigma$ are relative permeability, free-space permeability, permittivity, and conductivity, respectively; $\mathbf{J}$ is the current source; $V$ is the computational domain that encloses the circuit. A time-domain finite-element solution of (1) and its boundary condition results in a system of ordinary differential equations [14]

$$
\mathbf{T} \frac{d^{2} u}{d t^{2}}+\mathbf{R} \frac{d u}{d t}+\mathbf{S} u=\tilde{j}
$$

Driven by the continuous scaling of feature sizes and frequency enabled by the advancement in processing technology, in the past three decades, on-chip circuit modeling has experienced a series of transitions: from R (resistance)-based, RC (resistance-capacitance)-based, distributed RC-based, RLC (resistance-inductance-capacitance)-based, to transmission-line-based, to full-wave electromagnetics-based analysis [1]-[11]. However, on-chip circuits present many modeling challenges that are less pronounced in traditional full-wave applications such as antennas, waveguides, and microwave circuits. These challenges include ultra-large problem sizes, large number of conductors, conductor loss, large aspect ratio, broadband, the presence of substrate, etc. [1]. Among these challenges, large problem size is the primary challenge. In [12], [13], a time-domain layered finite-element reduction-recovery (TD-LAFE-RR) method was proposed to solve large-scale IC design problems at high frequencies. This method rigorously reduces the matrix of a multilayer system to that of a single-layer system regardless of the original problem size. More importantly, the matrix reduction is achieved analytically, and hence the CPU and memory overheads are minimal. In addition, the reduced system matrix preserves the sparsity of the original system matrix. Numerical experiments have demonstrated four-orders-of-magnitude reduction in matrix factorization time. However, like many time-domain methods, the time step of the TD-LAFE-RR method is bounded from above by a certain value to ensure the stability of the time-marching process. When applied to general multilayer structures in which the layout structures are different in different layers, i.e., the conductor configuration is different in different layers, the time

Manuscript received November 19, 2007; revised July 04, 2008. Current version published March 20,2009. This work was supported in part by a grant from the Office of Naval Research under award N00014-06-1-0716 and in part by a grant from Intel Corporation.

The authors are with the School of Electrical and Computer Engineering, Purdue University, West Lafayette, IN 47907 USA (email: djiao@purdue.edu).

Color versions of one or more of the figures in this paper are available online at http://ieeexplore.ieee.org.

Digital Object Identifier 10.1109/TAP.2008.2011393 in which $\mathbf{T}, \mathbf{R}$, and $\mathbf{S}$ are square matrices, and $u$ and $\tilde{j}$ are column vectors. Their elements are given by

$$
\begin{aligned}
\mathbf{T}_{i j} & =\mu_{0} \varepsilon\left\langle\mathbf{N}_{i}, \mathbf{N}_{j}\right\rangle_{V} \\
\mathbf{R}_{i j} & =\mu_{0} \sigma\left\langle\mathbf{N}_{i}, \mathbf{N}_{j}\right\rangle_{V} \\
\mathbf{S}_{i j} & =\mu_{r}^{-1}\left\langle\nabla \times \mathbf{N}_{i}, \nabla \times \mathbf{N}_{j}\right\rangle_{V} \\
\tilde{j}_{i} & =-\mu_{0}\left\langle\mathbf{N}_{i}, \partial_{t} \mathbf{J}\right\rangle_{V}
\end{aligned}
$$

where $\mathbf{N}_{i}$ are the vector bases used to expand the unknown electric field $\mathbf{E}$, and $\langle., .\rangle_{V}$ denotes volume integration. In (2), the matrix resulted from the absorbing boundary condition is omitted for simplicity. It can be easily incorporated into the framework after the core flow is established.

Adopting a central difference scheme to approximate the first- and second-order time derivative in (2), we obtain

$$
\mathbf{P} u^{n+1}=\left(2 \mathbf{T}-\Delta t^{2} \mathbf{S}\right) u^{n}+[0.5 \Delta t \mathbf{R}-\mathbf{T}] u^{n-1}+j^{n}
$$

in which $\mathbf{P}$ is the system matrix to be factorized

$$
\mathbf{P}=\mathbf{T}+0.5 \Delta t \mathbf{R}
$$

and $\Delta t$ represents the time step. The field value at the $(n+1)$-th time step, $u^{n+1}$, can be solved in a time marching fashion from the solution of $u$ at previous time steps. When the problem size is large, it is difficult to solve matrix (4). The time-domain LAFE-RR method [12], [13] was developed to overcome this problem. In this method, the 3D layered system matrix is analytically reduced to a $2 \mathrm{D}$ layered one. The 2D layered system matrix is further analytically reduced to a single-layer one. Once the unknowns on a single surface are known, the unknowns on other surfaces and the unknowns in the volume can be recovered as demonstrated in [12], [13].

To make the analytical reduction in the LAFE-RR scheme feasible for general multilayered structures, matrix $\mathbf{R}$, which is associated with conductivity $\sigma$ as shown in (3), needs to be excluded from the system 
matrix $\mathbf{P}$. This is because LAFE-RR method utilizes the layered property to perform system reduction analytically. In a general multilayered structure, permittivity is layered. However, conductivity is not layered. Hence, matrix $\mathbf{R}$ needs to be excluded from $\mathbf{P}$, i.e., $\mathbf{R}$ can only be present in the right hand side of (4).

Rewriting (2) as

$$
\mathbf{T} \frac{d^{2} u}{d t^{2}}+\mathbf{S} u=-\mathbf{R} \frac{d u}{d t}+j .
$$

Adopting a backward differencing scheme to approximate the firstorder time derivative, and a central differencing scheme to approximate the second-order time derivative in (6), we obtain

$$
\mathbf{T} \frac{u^{n+1}-2 u^{n}+u^{n-1}}{\Delta t^{2}}+\mathbf{S} u^{n}=-\mathbf{R} \frac{u^{n}-u^{n-1}}{\Delta t}+j^{n} .
$$

Performing a $z$-transform on (7), we obtain

$$
\mathbf{T} \frac{z^{2}-2 z+1}{\Delta t^{2}} \tilde{u}+\mathbf{S} z \tilde{u}=-\mathbf{R} \frac{z-1}{\Delta t} \tilde{u} .
$$

which can be rewritten as

$$
(z-1)^{2} \tilde{u}+\mathbf{T}^{-1} \mathbf{R}(z-1) \Delta t \tilde{u}+\Delta t^{2} \mathbf{T}^{-1} \mathbf{S} z \tilde{u}=0 .
$$

As a worst-case stability analysis, assuming the computational domain is uniformly filled by material of conductivity $\sigma$ and permittivity $\epsilon_{0}$, (9) becomes

$$
-\frac{(z-1)^{2}+\frac{\sigma \Delta t}{\varepsilon_{0}}(z-1)}{z} \tilde{u}=\Delta t^{2} \mathbf{T}^{-1} \mathbf{S} \tilde{u} .
$$

Denoting the eigenvalue of $\Delta t^{2} \mathbf{T}^{-1} \mathbf{S}$ by $\lambda$, we obtain

$$
(z-1)^{2}+\frac{\sigma \Delta t}{\varepsilon_{0}}(z-1)+\lambda z=0 .
$$

To make $z$ bounded in a unit circle to maintain the stability, the maximum allowed $\lambda$ is

$$
\lambda_{\max }=4-2 \frac{\sigma \Delta t}{\varepsilon_{0}} .
$$

Since $\lambda$ is the eigenvalue of $\Delta t^{2} \mathbf{T}^{-1} \mathbf{S}$, and the maximum modulus of the eigenvalues of $\Delta t^{2} \mathbf{T}^{-1} \mathbf{S}$ is $\rho\left(\Delta t^{2} \mathbf{T}^{-1} \mathbf{S}\right)$ while $\lambda$ is bounded from above by $\lambda_{\max }, \Delta t$ should satisfy the following criterion to ensure the stability of the LAFE-RR method

$$
\Delta t \leq \frac{\sqrt{\lambda_{\max }}}{\sqrt{\rho\left(\mathbf{T}^{-1} \mathbf{S}\right)}}
$$

in which $\rho(\bullet)$ denotes the spectral radius of matrix $(\bullet)$. As can be seen from (12), the larger the conductivity, and the smaller the permittivity, the smaller $\lambda_{\max }$, and hence the smaller the time step $\Delta t$. That's why the assumption of filling the computational domain uniformly by a material of conductivity $\sigma$ and permittivity $\epsilon_{0}$ constitutes a worst-case stability analysis of the right-R-based LAFE-RR scheme. In reality, the computational domain is partially filled by conductors. Also, the material has a larger permittivity than the permittivity of air, and hence the effective conductivity is smaller, and the effective permittivity is larger than what is considered in the worst-case analysis.

Since $\lambda_{\max }$ needs to be greater than 0 to render a time-marching scheme stable, from (12), it can be seen that

$$
\Delta t<\frac{2 \varepsilon_{0}}{\sigma} .
$$

The right-R-based LAFE-RR scheme was tested on typical coppermade on-chip structures. It was shown that $10^{-19} \mathrm{~s}$ is needed to main- tain the stability of the right-R-based LAFE-RR scheme. This agrees with our theoretical analysis in (14).

In (7), a backward differencing is used to approximate the first-order time derivative associated with $\mathbf{R}$. Certainly, other differencing schemes can be utilized as long as $\mathbf{R}$ is not associated with the most advanced time step, and hence is excluded from the system matrix to be factorized. A variety of differencing schemes were explored to approximate the first-order time derivative. It was observed that as long as $\mathbf{R}$ is excluded from the system matrix, the improvement in the maximum allowed time step is small.

If $\mathbf{R}$ is kept on the left hand side as in (2) and (5), the maximum allowed time step is still

$$
\Delta t \leq \frac{\sqrt{\lambda_{\max }}}{\sqrt{\rho\left(\mathbf{T}^{-1} \mathbf{S}\right)}}
$$

but $\lambda_{\max }$ is equal to 4 . This leads to a much larger time step. Testing on typical on-chip structures has shown that the time step at a level of $10^{-16} \mathrm{~s}$ can be used to maintain the stability, which is three orders of magnitude larger than $10^{-19} \mathrm{~s}$. However, if $\mathbf{R}$ is kept on the left hand side of (2), apparently, a LAFE-RR solution is not feasible because conductivity is not layered in a general multilayered structure. In the Section III, we describe a fast-marching method that enlarges the time step required by the LAFE-RR method by three orders of magnitude. In addition, it enlarges the time step without losing the computational efficiency of the LAFE-RR method.

\section{Proposed Fast-Marching Method}

We still keep $\mathbf{R}$ at the left hand side of (2). The matrix equation we solve in time domain is still (4), which can be rewritten as

$$
\begin{aligned}
& (\mathbf{T}+0.5 \Delta t \mathbf{R}) u^{n+1} \\
& \quad=\left(2 \mathbf{T}-\Delta t^{2} \mathbf{S}\right) u^{n}+[0.5 \Delta t \mathbf{R}-\mathbf{T}] u^{n-1}+j^{n} .
\end{aligned}
$$

If (15) is solved in time instead of (7), a three-orders-of-magnitude increase in the time step can be achieved. Therefore, as long as (15) is solved using the LAFE-RR method, the time step can be increased without losing the computational efficiency. The task is hence narrowed down to the development of a LAFE-RR solution for (15).

To ease the illustration of the proposed method, we denote (15) by

$$
(\mathbf{T}+0.5 \Delta t \mathbf{R}) x=b .
$$

Equation (16) is solved at each time step in the time marching process. To enable a LAFE-RR solution, $\mathbf{R}$ is split into two parts as

$$
\mathbf{R}=\mathbf{R}_{c}-\mathbf{R}_{0}
$$

in which $\mathbf{R}_{c}$ is artificially constructed to permit a LAFE-RR solution, and $\mathbf{R}_{\circ}$ is the difference between $\mathbf{R}_{c}$ and $\mathbf{R}$. Equation (16) at each time step is then solved iteratively by

$$
\begin{aligned}
\left(\mathbf{T}+0.5 \Delta t \mathbf{R}_{c}\right) x^{(k+1)} & =b+0.5 \Delta t \mathbf{R}_{0} x^{(k)} \\
k & =0,1,2, \ldots
\end{aligned}
$$

It is clear that when the convergence is reached, $x^{(k)}=x^{(k+1)}$, and hence $x^{(k)}$ is the solution of (16). In (18), the initial value $x^{(0)}$ can be chosen as

$$
x^{(0)}=u^{n}
$$

which is the field value at the previous time step; it can also be chosen as

$$
x^{(0)}=2 u^{n}-u^{n-1}
$$


which is linearly extrapolated from the field values at the previous two time steps. Both choices work effectively. But (20) is shown to produce a better accuracy by our numerical experiments. Next, we will explain the proposed fast-marching method from three perspectives: the construction of $\mathbf{R}_{c}$, the convergence of (18), and the LAFE-RR solution of (15).

\section{A. The Construction of $\mathbf{R}_{c}$}

$\mathbf{R}_{c}$ in (17) is a conductivity related matrix. It is assembled from its elemental contribution as

$$
\mathbf{R}_{c, i j}^{e}=\mu_{0} \sigma_{c}^{e}\left\langle\mathbf{N}_{i}, \mathbf{N}_{j}\right\rangle_{V}
$$

in which $\sigma_{c}^{e}$ is artificially assigned conductivity in element $e$, which is determined based on

$$
\sigma_{c}^{e}= \begin{cases}\sigma^{e} & \text { if } \sigma^{e}>0 \\ \sigma \text { or } 0 & \text { if } \sigma^{e}=0\end{cases}
$$

in which $\sigma^{e}$ is the true (physical) conductivity in element $e$. In elements that $\sigma^{e}$ is $0, \sigma_{c}^{e}$ is chosen as either $\sigma$ (nonzero) or 0 so that when adding $\mathbf{R}_{c}$ upon $\mathbf{T}$, the combined matrix permits a LAFE-RR solution.

To construct $\mathbf{R}_{c}$, one approach is to introduce a solid metal plane in each metal layer. This will allow the matrix combined from $\mathbf{R}_{c}$ and $\mathbf{T}$ to permit a LAFE-RR solution for any multilayered structure. For example, for a RF IC circuit, $\sigma_{c}$ is chosen as the metal conductivity in all the metal layers, while it is set to 0 in the other dielectric layers. As a result, in each layer along the $z$ direction (stack-growth direction), conductivity is a constant. Hence, when combining $\mathbf{R}_{c}$ and $\mathbf{T}$ together, the resultant matrix permits a LAFE-RR solution in which the reduction from a 3D layered system to a single-layer one can be achieved analytically without any computational cost. As another example, for an on-chip interconnect network in which the layer-growth direction is often chosen as $x$ or $y$, by introducing a solid plane in each metal layer to construct $\mathbf{R}_{c}$, the configuration of conductivity is the same for each layer along either $x$-or $y$-direction. As a result, when combining $\mathbf{R}_{c}$ and $\mathbf{T}$ together, the resultant system matrix also permits a LAFE-RR solution. Introducing a solid plane in each metal layer is the simplest and most generalized approach to construct $\mathbf{R}_{c}$. Depending on the structure, one could select other $\mathbf{R}_{c} \mathrm{~s}$ as long as the conductivity distribution permits a LAFE-RR solution.

\section{B. The Convergence of the Iterative Scheme in (18)}

In [16], the following theorem was proven.

Theorem 1: Define a sequence of iterates of the form

$$
x^{(k+1)}=\mathbf{G} x^{(k)}+f
$$

Let $\mathbf{G}$ be a square matrix such that $\rho(\mathbf{G})<1$, then $\mathbf{I}-\mathbf{G}$ is nonsingular and the iteration in (23) converges for any initial vector $x^{(0)}$.

From (18), it can be seen that $\mathbf{G}$ in our iteration scheme is

$$
\mathbf{G}=\left(\mathbf{T}+0.5 \Delta t \mathbf{R}_{c}\right)^{-1}\left(0.5 \Delta t \mathbf{R}_{0}\right) .
$$

Denoting the eigenvalues of $\mathbf{G}$ by $\lambda$, we have

$$
\mathbf{G} x=\lambda x
$$

in which $x$ denotes the eigenvector. From (24) and (25), we obtain

$$
\left(0.5 \Delta t \mathbf{R}_{0}\right) x=\lambda\left(\mathbf{T}+0.5 \Delta t \mathbf{R}_{c}\right) x
$$

$\mathbf{R}_{0}$ can be assembled from elemental contribution as

$$
\mathbf{R}_{0}=\left[\mu_{0} \sigma_{0}^{e}\left\langle\mathbf{N}_{i}, \mathbf{N}_{j}\right\rangle_{V}\right]
$$

in which

$$
\sigma_{0}^{e}=\sigma_{c}^{e}-\sigma^{e}
$$

as can be seen from (17) and (21). From (22), we obtain

$$
\sigma_{0}^{e} \leq \sigma_{c}^{e} .
$$

Equation (26) can be written as

$$
0.5 \Delta t \mu_{0}\left[\sigma_{0}^{e}\left\langle\mathbf{N}_{i}, \mathbf{N}_{j}\right\rangle_{V}\right] x=\lambda\left[\left(\mu_{0} \varepsilon^{e}+0.5 \Delta t \mu_{0} \sigma_{c}^{e}\right)\left\langle\mathbf{N}_{i}, \mathbf{N}_{j}\right\rangle_{V}\right] x
$$

in which $[\cdot]$ denotes an assembling from the elemental contribution. Take a RF IC circuit as an example and consider a single layer, $\epsilon$ and $\sigma_{c}$ are constant whereas $\sigma_{0}$ is space dependent. The eigenvalue $\lambda$ can be calculated from

$$
\lambda=\frac{0.5 \Delta t \mu_{0} x^{T}\left[\sigma_{0}^{e}\left\langle\mathbf{N}_{i}, \mathbf{N}_{j}\right\rangle_{V}\right] x}{\left(\mu_{0} \varepsilon+0.5 \Delta t \mu_{0} \sigma_{c}\right) x^{T}\left[\left\langle\mathbf{N}_{i}, \mathbf{N}_{j}\right\rangle_{V}\right] x}
$$

By replacing $\sigma_{0}^{e}$ in each element uniformly by $\sigma_{c}$, we can obtain the upper bound of $\lambda$ 's modulus

$$
|\lambda|<\frac{0.5 \Delta t \mu_{0} \sigma_{c}\left|x^{T}\left[\left\langle\mathbf{N}_{i}, \mathbf{N}_{j}\right\rangle_{V}\right] x\right|}{\left(\mu_{0} \varepsilon+0.5 \Delta t \mu_{0} \sigma_{c}\right)\left|x^{T}\left[\left\langle\mathbf{N}_{i}, \mathbf{N}_{j}\right\rangle_{V}\right] x\right|} .
$$

Clearly

$$
|\lambda|<1
$$

Therefore, the condition of $\rho(\mathbf{G})<1$ is satisfied.

In general cases, $\epsilon, \sigma_{c}$, and $\sigma_{0}$ are all space dependent. To theoretically analyze the upper bound of the eigenvalues of $\mathbf{G}$, we can approximate the left matrix in (30) by

$$
0.5 \Delta t \mu_{0}\left[\sigma_{0}^{e}\left\langle\mathbf{N}_{i}, \mathbf{N}_{j}\right\rangle_{V}\right] \sim 0.5 \Delta t \mu_{0} \sigma_{0, \mathrm{eff}}\left[\left\langle\mathbf{N}_{i}, \mathbf{N}_{j}\right\rangle_{V}\right]
$$

in which an effective $\sigma_{0}, \sigma_{0, \text { eff }}$, is used to fill every element. Clearly, $\sigma_{0, \text { eff }}$ is bounded between the minimum $\sigma_{0}$ and the maximum $\sigma_{0}$. Similarly, the right matrix in (30) can be approximated by

$$
\begin{aligned}
{\left[\left(\mu_{0} \varepsilon^{e}+0.5 \Delta t \mu_{0} \sigma_{c}^{e}\right)\right.} & \left.\left\langle\mathbf{N}_{i}, \mathbf{N}_{j}\right\rangle_{V}\right] \\
& \sim\left(\mu_{0} \varepsilon_{\mathrm{eff}}+0.5 \Delta t \mu_{0} \sigma_{c, \mathrm{eff}}\right)\left[\left\langle\mathbf{N}_{i}, \mathbf{N}_{j}\right\rangle_{V}\right]
\end{aligned}
$$

in which effective conductivity and effective permittivity, $\sigma_{0, \text { eff }}$ and $\epsilon_{\mathrm{eff}}$, are used to fill every element. As a result, $\lambda$ can be estimated from

$$
|\lambda| \sim \frac{0.5 \Delta t \mu_{0} \sigma_{0, \mathrm{eff}}\left|x^{T}\left[\left\langle\mathbf{N}_{i}, \mathbf{N}_{j}\right\rangle_{V}\right] x\right|}{\left(\mu_{0} \varepsilon_{\mathrm{eff}}+0.5 \Delta t \mu_{0} \sigma_{c, \mathrm{eff}}\right)\left|x^{T}\left[\left\langle\mathbf{N}_{i}, \mathbf{N}_{j}\right\rangle_{V}\right] x\right|} .
$$

Since in every element, $\sigma_{0}^{e} \leq \sigma_{c}^{e}$ we have

$$
\sigma_{0, \mathrm{eff}} \leq \sigma_{c, \mathrm{eff}} .
$$

Hence, from (36), it can be deduced that $|\lambda|<1$. As a result, $\rho(\mathbf{G})<1$ is satisfied for general cases also. Hence, the iterative scheme in (18) always converges regardless of the choice of the initial vector. Also, since at each time step, the previous values such as (19) and (20) are used as the initial guess, the iteration can converge very rapidly.

\section{The LAFE-RR Solution}

Since $\left(\mathbf{T}+0.5 \Delta t \mathbf{R}_{c}\right)$ permits a LAFE-RR solution, the following LAFE-RR steps are taken to solve (15).

Step 1: Analytically reduce the multilayer system matrix of $(\mathbf{T}+$ $0.5 \Delta t \mathbf{R}_{c}$ ) to a single-layer system matrix. 


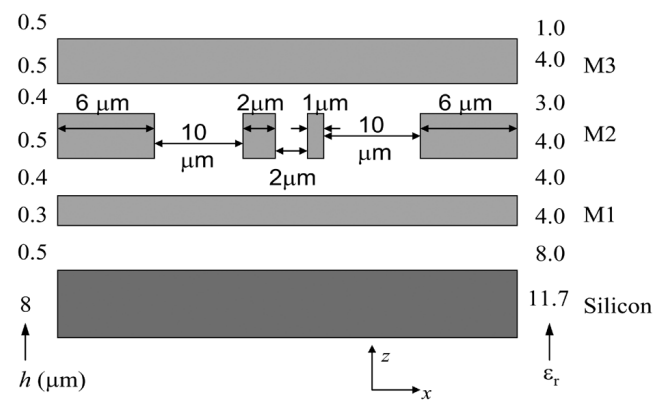

(a)

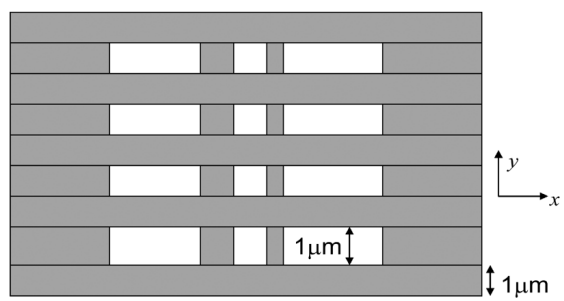

(b)

Fig. 1. Geometry of a three-metal-layer on-chip interconnect. (a) Cross-sectional view. (b) Top view.

Step 2: Pre-calculate the LU factorization of the single-layer system matrix for the time marching process.

Beginning of the Time Marching:

Step 3: Perform the iteration in (18). When the iteration converges, $u^{n+1}$ in (15) is obtained.

Sub-step 1: Solve the single-layer system and obtain surface unknowns in that single layer.

Sub-step 2: Recover solutions of surface and volume unknowns in all other layers.

Step 4: Construct the new right hand side of (15) for the next time step. Go back to step 3.

End of the Time Marching.

Since the number of iterations to reach a required accuracy in Step 3 is very small ( 1 or 2 on average in our numerical experiments), the proposed method increases the time step without losing the computational efficiency of the original LAFE-RR method.

Before proceeding to the numerical validation, it is worth mentioning that (15) can also be solved by using other iterative solvers such as conjugate gradient (CG) method with $\left(\mathbf{T}+0.5 \Delta t \mathbf{R}_{c}\right)$ chosen as the preconditioner. Since both $\left(\mathbf{T}+0.5 \Delta t \mathbf{R}_{c}\right)$ and $(\mathbf{T}+0.5 \Delta t \mathbf{R})$ are symmetric positive definite. The convergence of $\mathrm{CG}$ is also guaranteed. In addition, the solution of $\left(\mathbf{T}+0.5 \Delta t \mathbf{R}_{c}\right)$ is computationally cheap by using the LAFE-RR method.

\section{NUMERICAL AND EXPERIMENTAL VALIDATION}

The first example considered here is a three-metal-layer on-chip interconnect structure. The geometry is depicted in Fig. 1 from both the cross-sectional view (in $x-z$ plane) and the top view (in $x-y$ plane). All the dimensions are close to realistic on-chip circuits. The conductivity of all metals is $5 \times 10^{7} \mathrm{~S} / \mathrm{m}$. The conductivity of the silicon substrate is $10^{4} \mathrm{~S} / \mathrm{m}$. There exist five $x$-orientated wires in M1 and M3 layers, respectively, as shown in Fig. 1(b). The width of these wires is $1 \mu \mathrm{m}$, and the spacing is $1 \mu \mathrm{m}$. The interconnect length $(9 \mu \mathrm{m})$ along $y$ is subdivided into 9 layers. Its two ends are both attached to an air layer, which is truncated by a first-order absorbing boundary condition. The top and bottom boundaries along $z$-direction are PEC (perfect electrically conducting) boundaries. The left and right boundaries along $x$-direction are PMC (perfect magnetically conducting) boundaries. The layer growth direction is chosen as $y$. Each layer is divided into 780 triangular prism elements.

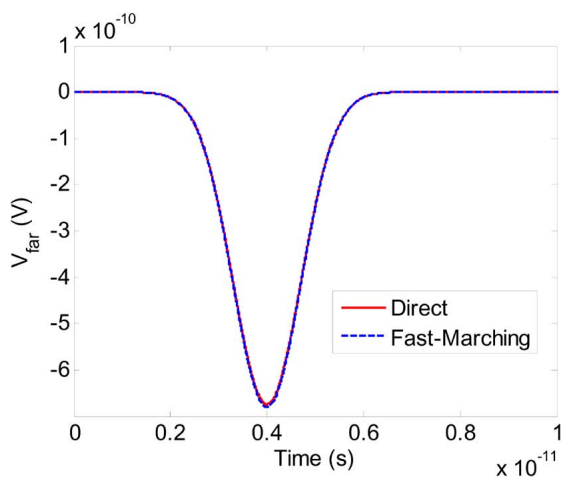

Fig. 2. The voltage simulated by the proposed method in comparison with a direct simulation.

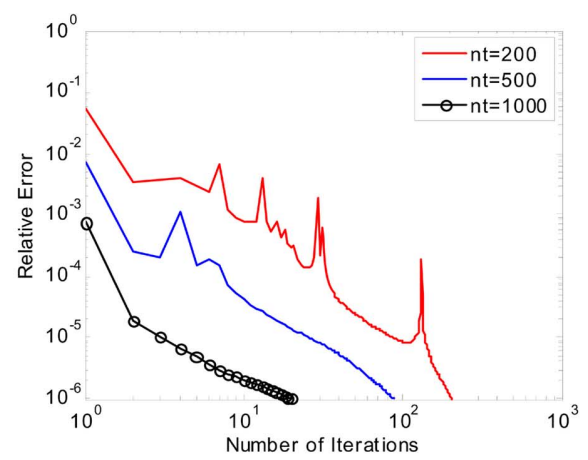

(a)

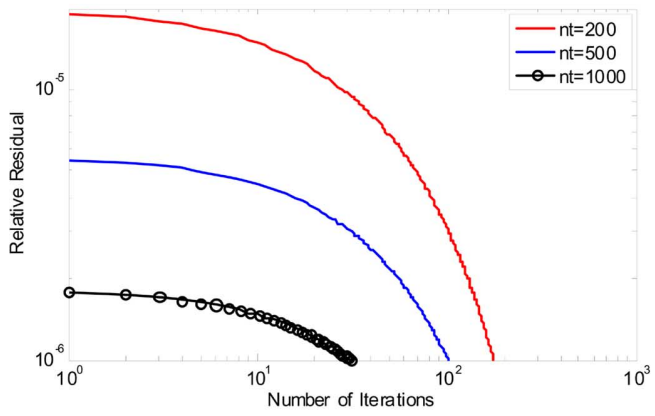

Fig. 3. Illustration of convergence. (a) Relative error versus number of iterations. (b) Relative residual versus number of iterations.

Since the configuration of conductivity is different in different layers along $y$, using the original LAFE-RR method, the right-R-based scheme is adopted, which requires a time step as small as $10^{-19} \mathrm{~s}$ to maintain stability. By using the proposed fast-marching method, the time step is significantly increased to $10^{-16} \mathrm{~s}$ while still maintaining the stability of the time marching process. The matrix $\mathbf{R}_{c}$ for this example is constructed by replacing the interconnect wires in M1 and M3 layers by solid planes while keeping the conductors in M2 layer the same. When iteratively solving (18), the relative error for the convergence checking is defined as

$$
\delta=\frac{1}{N} \sum_{i=1}^{N} \frac{\left|x_{i}^{(k+1)}-x_{i}^{(k)}\right|}{\left|x_{i}^{(k)}\right|}
$$

in which $N$ is the dimension of the unknown field vector. As can be seen from (18), when the difference between $x^{(k)}$ and $x^{(k+1)}$ shrinks to zero, $x^{(k+1)}$ becomes the solution of (16). Hence, the relative error defined in (38) can serve as a good measure of convergence. It is also computationally inexpensive compared to relative residual that involves the computation of a large matrix-vector multiplication. 

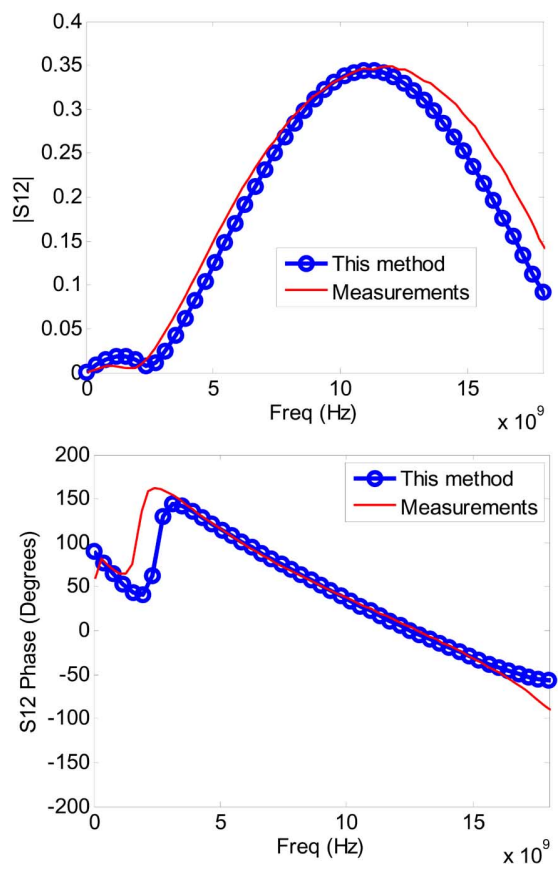

Fig. 4. Crosstalk simulation of a large-scale on-chip 3D interconnect.

The near end of the $1 \mu \mathrm{m}$-wide wire in $\mathrm{M} 2$ was excited by a current source. The source waveform was the time derivative of a Gaussian pulse, $\mathbf{J}^{\text {inc }}(t)=\hat{x} 2\left(t-t_{0}\right) \exp \left[-\left(t-t_{0}\right)^{2} / \tau^{2}\right]$, in which $\tau$ is $1.0 \mathrm{e}-12 \mathrm{~s}$, and $t_{0}$ is $4 \tau$. The current probe was placed underneath the $1 \mu \mathrm{m}$-wide wire in the dielectric layer between M1 layer and M3 layer. The voltage at the far end of the $1 \mu \mathrm{m}$-wide wire was simulated. In Fig. 2, the result obtained by the proposed fast marching method is compared with that obtained by solving (15) directly without using the LAFE-RR method. As shown in Fig. 2, an excellent agreement was observed, which demonstrates the accuracy of the proposed method.

The maximum allowed relative error was set to 0.01 for the numerical simulation that produces the result shown in Fig. 2. In total, 100,000 time steps were run. The number of iterations was $\sim 30$ at the beginning, and very soon dropped to 1 . The average number of iterations was 1 over the 100,000 time steps. Hence, the time step was increased without sacrificing the computational efficiency of the original LAFE-RR method. In Fig. 3(a), the relative error is plotted with respect to the number of iterations at $t=200 \Delta t, t=500 \Delta t$, and $t=1000 \Delta t$, respectively. Fast convergence was observed. The relative residual of (18) and (16) with respect to the number of iterations is also plotted in Fig. 3(b). The relative residual is defined as $\|(\mathbf{T}+0.5 \Delta t \mathbf{R}) x-b\| /\|b\|$, in which 2 -norm is used. It can be seen that the relative error defined in (38) turns out to be a more stringent convergence criterion compared to the relative residual in this scenario.

The second example is a large-scale test-chip interconnect structure that involves 2000 orthogonal wires in M1 and M3 layers. Due to a nondisclosure agreement, the detail of this structure is not given here. The matrix $\mathbf{R}_{c}$ for this example is constructed by placing a solid metal plane in M1 and M3 layers respectively, while keeping M2 conductors the same. The voltages are sampled at the near ends of two M2 wires, the far ends of which are left open. The crosstalk of the two near-end ports are then extracted and compared with the measured data. As shown in Fig. 4, good agreement was observed. Note that a time step of $10^{-16} \mathrm{~s}$ was used in this simulation, which cannot be achieved by the original right-R-based LAFE-RR scheme. The number of iterations at each time step was 1 on average in this simulation.

\section{CONCLUSION}

In this work, the time step allowed by a TD-LAFE-RR method in general on-chip applications was analyzed. A fast-marching method was developed to increase the time step by three orders of magnitude, while maintaining the same computational efficiency of the original LAFE-RR method. Numerical and experimental results demonstrated the validity of the proposed method.

\section{ACKNOWLEDGMENT}

The authors would like to thank Dr. M. J. Kobrinsky and Dr. S. Chakravarty at Intel Corporation for providing measured data.

\section{REFERENCES}

[1] D. Jiao, C. Dai, S.-W. Lee, T. R. Arabi, and G. Taylor, "Computational electromagnetics for high-frequency IC design," in Proc. IEEE Int. Symp. on Antennas and Propag., 2004, pp. 3317-3320.

[2] P. J. Restle, A. E. Ruehli, S. G. Walker, and G. Papadopoulos, "Fullwave PEEC time-domain method for the modeling of on-chip interconnects," IEEE Trans. Comput.-Aided Design, vol. 20, no. 7, pp. 877-887, Jul. 2001

[3] A. Rong, A. C. Cangellaris, and L. Dong, "Comprehensive broadband electromagnetic modeling of on-chip interconnects with a surface discretization-based generalized PEEC model," in Proc. IEEE 12th Topical Meeting on Elect. Perform. of Electron. Pckg. (EPEP), 2003, pp. 367-370.

[4] Z. H. Zhu, B. Song, and J. K. White, "Algorithms in fastimp: A fast and wideband impedance extraction program for complicated 3D geometries," IEEE Trans. Comput.-Aided Design, vol. 24, no. 7, pp. 981-998, Jul. 2005.

[5] D. Jiao, M. Mazumder, S. Chakravarty, C. Dai, M. Kobrinsky, M. Harmes, and S. List, "A novel technique for full-wave modeling of large-scale three-dimensional high-speed on/off-chip interconnect structures," in Proc. Int. Conf. on Simulation of Semicond. Processes and Devices, 2003, pp. 39-42.

[6] S. Kapur and D. E. Long, "Large-scale full-wave simulation," in Proc. 41th ACM/IEEE Design Automat. Conf. (DAC), 2004, pp. 806-809.

[7] D. Gope, A. E. Ruehli, C. Yang, and V. Jandhyala, "(S)PEEC: Time-and frequency-domain surface formulation for modeling conductors and dielectrics in combined circuit electromagnetic simulations," IEEE Trans. Microw. Theory Tech., vol. 54, no. 6, pp. 2453-2464, Jun. 2006.

[8] Z. G. Qian, J. Xiong, L. Sun, I. T. Chiang, W. C. Chew, L. J. Jiang, and Y. H. Chu, "Crosstalk analysis by fast computational algorithms," in Proc. IEEE 14th Topical Meeting on Elect. Performance Electron. Packag., 2005, pp. 367-370.

[9] F. Ling, V. I. Okhamtovski, W. Harris, S. McCracken, and A. Dengi, "Large-scale broadband parasitic extraction for fast layout verification of 3D RF and mixed-signal on-chip structures," IEEE Trans. Microw. Theory Tech., vol. 53, no. 1, pp. 264-273, Jan. 2005.

[10] D. Jiao, S. Chakravarty, and C. Dai, "A layered finite-element method for high-capacity electromagnetic analysis of high-frequency ICS," IEEE Trans. Antennas Propag., vol. 55, pp. 422-432, Feb. 2007.

[11] Z. Y. Yuan, Z. F. Li, and M. L. Zou, "Computer-aided analysis of on-chip interconnects near semiconductor substrate for high-speed VLSI," IEEE Trans. Comput.-Aided Design, vol. 19, no. 9, pp. 990-998, Sep. 2000.

[12] H. Gan and D. Jiao, "A time-domain layered finite element reduction recovery (LAFE-RR) method for high-frequency VLSI design," IEEE Trans. Antennas Propag., vol. 55, pp. 3620-3629, Dec. 2007.

[13] H. Gan and D. Jiao, "A fast and high-capacity electromagnetic solution for high-speed IC design," in Proc. IEEE/ACM Int. Conf. Comput.Aided Design (ICCAD), 2007, pp. 1-6.

[14] D. Jiao and J. M. Jin, "Finite element analysis in time domain," in The Finite Element Method in Electromagnetics. New York: Wiley, 2002, pp. 529-584.

[15] D. Jiao and J. M. Jin, "A general approach for the stability analysis of time-domain finite element method," IEEE Trans. Antennas Propag., vol. 50, pp. 1624-1632, Nov. 2002.

[16] Y. Saad, Iterative Methods for Sparse Linear Systems, 2nd ed. Philadelphia, PA: SIAM, 2003. 